\title{
Complément à la connaissance de la morphologie et de la biologie de Proteocephalus macrocepbalus (Creplin 1825), cestode parasite de l'Anguille
}

\author{
Par J.-M. DOBY et L. JARECKA *
}

Il est fréquent, chez les Salmonidés par exemp.e, qu'une même espèce de poisson puisse héberger plusieurs espèces de Cestodes du genre Proteocephalus. Par contre, chez l'anguille commune, Anguilla anguilla (= Anguilla vulgaris), un seul Cestode de cette famille digne d'être retenu a été signalé (1), Proteocephalus macrocephalus (Creplin $1825)$; ce Cestode, qui a une aire de répartition géographique considérable, puisqu'il se rencontre aussi bien dans le Nouveau continent que dans l'Ancien, peut parasiter d'autres espèces d'anguilles (Anguilla chrysipa et Anguilla rostrata).

La morphologie de Proteocephalus macrocephalus à l'état adulte a fait l'objet de plusieurs descriptions (Schneider, 1905, La Rue, 1914, etc...). Sa détermination spécifique, si l'on se réfère aux difficultés rencontrées dans l'identification de la plupart des autres espèces de cette famille (Doby et Jarecka, 1964), est relativement facile.

Nous avons eu l'occasion de trouver ce Cestode en assez grande abondance chez des anguilles adultes en provenance des bords de la Loire (région de Nantes et SaintNazaire). Les caractères des exemplaires que nous avons réco!tés correspondent assez bien à ceux donnés par les auteurs précédents (dimensions générales, dimensions des ventouses principales, nombre de testicules, rapport *longueur de la poche du pénis

(1) En effet, une autre espèce, Proteocephalus longicollis (Zeder, 1800), parasite habituel de Salmonidés, a été signalée chez l'anguille dans le lac de Lucerne par Nufer (1905). Toutefois, pour La Rue (1914), des doutes sont à émettre concernant cette observation. L'opinion de ce dernier semble justifiée quand on connaît la relative spécificité de ces Cestodes.

Par ailleurs, deux autres espèces de Protéocéphales ont été décrites chez Anguilla vulgaris: Proteocephalus dilatatus (Linton, 1889) et Proteocephalus hemisphericus (Molin, 1859). La première de ces deux espèces, qui a été décrite d'Amérique du Nord (in Yamaguti, 1959), ne figure pas dans l'ouvrage de Wardle et Mc Leod (1952). Quant à la seconde, décrite d'Europe, elle est considérée par les deux auteurs précités comme \&species inquirenda».

(*) Présenté à la Société française de Parasitologie dans sa séance du 27 novembre 1965.

Annales de Parasitologie humaine et comparée (Paris), t. 41, 1966, $\mathrm{n}^{\circ} 5$ 
sur largeur totale de l'anneau »), compte tenu cependant des variations imputables à l'état de plus ou moins grande contraction à l'état frais, ou, après fixation, selon la compression subie par le cestode entre lame et lamelle.

Aussi, nous n'estimons pas utile d'en refaire ici la description complète. Les seuls caractères sur lesquels nous nous contenterons d'insister, caractères non décrits antérieurement ou objets de descriptions parfois contradictoires, sont respectivement la $5^{\circ}$ ventouse, le revêtement cuticulaire, les dimensions du pénis et l'œuf :

\section{a) La $5^{\circ}$ ventouse :}

Voici le diamètre donné par les auteurs précités : $25 \mu$ (Schneider) ; 20 à $25 \mu$ (LaRue - pour cet auteur, il ne s'agit pas en fait d'une ventouse, mais seulement d'un massif cellulaire) ; $27 \mu$ (Markowski, 1933 - «très faiblement développée »). Le caractère rudimentaire de la $5^{\circ}$ ventouse est repris ultérieurement dans les descriptions de nombreux auteurs (Joyeux et Baer (1936): * ventouse apicale rudimentaire et ne mesurant que $25 \mu$ de diamètre » - et par Wardle et Mc Leod (1952) : «apical suker vestigial »).

Sans doute par l'étude d'un matériel à frais en très bonnes conditions et grâce à l'utilisation du contraste de phase, nous-mêmes avons pu observer une $5^{\circ}$ ventouse particulièrement nette, souvent même creusée d'une dépression apparemment fonctionnelle et dont voici les dimensions :

— diamètre extérieur $40 \mu$; diamètre de l'ouverture $20 \mu$ (matériel à frais, non comprimé, ou à peine, entre lame et lamelle).

\section{b) Le revêtement cuticulaire :}

La cuticule, sur toute la longueur du Cestode, est très finement spinulée, cette spinulation n'étant visible qu'à frais et en contraste de phase.

\section{c) Le pénis :}

Cet organe, que nous avons eu la chance d'observer partiellement en extrusion, mesure au moins $150 \mu$ de long sur un diamètre à la base de 80 à $90 \mu$. Le premier de ces chiffres est vraisemblablement très inférieur à la réalité si l'on tient compte de la présence, sur la face intérieure de la lumière des pénis que nous avons observés, d'une spinulation identique à celle observée extérieurement sur la cuticule, ce qui laisse à penser que cette extrusion n'était pas totale.

\section{d) L'œuf :}

En plaçant simplement dans de l'eau ordinaire les Cestodes adultes, nous avons pu obtenir la libération d'œufs à maturité.

Nous pensons utile d'en faire ici la description, les caractères donnés par les auteurs précités étant à notre avis peu valables, les dimensions notamment, car concernant des œufs non mûrs encore inclus dans l'utérus des Cestodes après fixation.

L'œuf mûr présente trois enveloppes : 
a) L'enveloppe la plus externe : Elle a un diamètre variant de 30 à $34 \mu$ (œuf non comprimé entre lame et lamelle). Elle est fine, lisse, incolore.

b) L'enveloppe médiane : Son diamètre est de 26 à $29 \mu$. Elle est tapissée intérieurement par des cellules.

c) La membrane interne : Elle est étroitement accolée à l'onchosphère dont voici les dimensions : 18 à $22 \mu$ pour le plus grand diamètre et 16 à $18 \mu$ pour le diamètre le plus faible.

Les six crochets embryonnaires ont les dimensions suivantes :

- crochets $c$ (médians) : 9 à $10 \mu$ de long ;

- crochets $b$ (latéro-internes) : 9 à $9,5 \mu$ de long ;

— crochets $a$ (latéro-externes) : 7,5 $\mu$ à $8 \mu$ de long.

La morphologie de ces différents crochets n'est pas identique. Les latéro-externes ont un manche considérablement plus mince que les autres. Leur lame. beaucoup plus effilée, est par contre relativement plus longue, puisqu'elle atteint $3 \mu$ alors que celles des autres crochets font environ $2,5 \mu$ (schéma).

\section{Infestations d'Hôtes intermédiaires}

Ces œufs à maturité nous ont permis de tenter l'infestation d'une douzaine d'espèces de Copépodes.

Dans la nature, une larve attribuée à Proteocephalus macrocephalus a été observée en Pologne par l'un de nous (Jarecka 1960). Cette identification cependant, avec le recul du temps, nous semble pouvoir être l'objet de réserves pour des raisons à la fois morphologiques et biologiques :

a) Chez cette larve, il n'y avait pas de $5^{\circ}$ ventouse visible, alors qu'elle est particulièrement nette, même à l'état larvaire, comme nous le verrons plus loin. Par ailleurs, la taille observée alors $(1300 \mu$ sur $400 \mu)$ dépasse de très loin celle des plus gros exemplaires à maturité que nous avons observés chez les diverses espèces de Copépodes expérimentalement réceptives. Même s'il est difficile de tenir compte d'une dimension larvaire en valeur absolue, comme nous en discuterons plus loin, les dimensions relatives du corps et des ventouses ne concordent pas avec celles de nos larves $(53 \mu$ sur $1.300 \mu$ et $70 \mu$ sur $950 \mu$ respectivement). Enfin, le tracé des canaux excréteurs, en tenant compte cependant de la difficulté de son observation, diffère assez sensiblement de celui des canaux excréteurs de nos larves.

b) La larve en question fut observée chez Cyclops insignis (4 individus parasités sur 169 examinés). Dans le même biotope furent examinés sans résultat 195 Acanthocyclops vernalis. Or, cette dernière espèce, entre nos mains, expérimentalement, s'est révélée particulièrement réceptive à l'infestation puisque nous avons obtenu un taux 
d'environ $70 \%$. Nous pensons que, statistiquement, un certain nombre d'Acanthocyclops vernalis eût dû être trouvé infesté lors des recherches effectuées sur le terrain.

Les infestations furent tentées en plaçant simplement les œufs mûrs obtenus comme décrit précédemment dans les récipients d'élevage des Copépodes, dans des conditions aussi naturelles que possible.

Parmi les douze espèces utilisées au cours de notre expérimentation, nous pouvons distinguer :

$1^{\circ}$ Celles chez lesquelles les onchosphères n'ont pas été retrouvées dans la cavité générale quelques heures après la mise en présence des Crustacés et des œufs mûrs, sans qu'il nous soit cependant possible de dire la raison de cette absence (ou bien non ingestion des œufs, ou bien, après ingestion, digestion des onchosphères, ou enfin, après ingestion, mais non digestion, élimination des onchosphères avec les matières fécales du Copépode après avoir subi sans dommage le transit intestinal).

- Macrocyclops albidus (Jurine 1820).

- Macrocyclops fuscus (Jurine 1820).

- Megacyclops viridis (Jurine 1820).

- Megacyclops gigas (Claus 1857).

Tous ces Copépodes provenaient de différentes collections d'eau de l'Ouest de la France (Ille-et-Vilaine et Loire-Atlantique).

$2^{\circ}$ Celles chez lesquelles les onchosphères ont été retrouvées bien vivantes dans la cavité générale, mais sans que ce passage ait été suivi de l'évolution de la larve pendant la quinzaine de jours d'observation après l'infestation :

- Eucyclops serrulatus (Fischer 1851).

- Mesocyclops leuckarti (Claus 1857).

Ces Copépodes avaient la même origine que les précédents.

$3^{\circ}$ Celles chez lesquelles l'évolution a pu être observée et suivie souvent jusqu'à la totale maturité de la larve.

Voici la liste des espèces, leur provenance, les taux d'infestation, l'intensité de ce.le-ci et la morphologie des larves de Protéocéphale qui y ont été observées au cours des divers prélèvements dans le temps (dimensions relevées sur des larves vivantes non ou très légèrement comprimées entre lame et lamelle).

Nous jugeons utile de donner séparément les caractères des larves ayant évolué dans chaque espèce. Comme nous le verrons en effet, d'une espèce de Copépode à l'autre, s'observent des différences considérables de rapidité d'évolution des larves de Protéocéphale. Malheureusement, pour aucune des espèces de Copépodes figurant dans cette liste n'a été effectuée une observation suivie de l'évolution larvaire à des intervalkes de temps réguliers.

Nous avons d'ailleurs constaté que chez une même espèce de Copépode, l'évolution des larves d'une même espèce de Protéocéphale pouvait varier considérablement et n'était pas toujours fonction de leur nombre dans la cavité générale, plusieurs ayant parfois paradoxalement une croissance plus rapide qu'une larve isolée. 
a) Cyclops strenuus strenuus (Fischer, 1851) (2)

Des Copépodes de cette espèce, récoltés depuis un certain temps dans des bassins de Pisciculture de la station d'Hydrobiologie de Thonon (I.N.R.A.), avaient déjà été infectés expérimentalement plusieurs semaines auparavant avec des œufs d'un Protéocéphale de corégone (Jarecka et Doby 1965) (3) et présentaient de ce fait déjà chacun plusieurs formes larvaires dans leur cavité générale. Ce polyparasitisme est la raison pour laquelle il ne nous est pas possible de donner ioi le pourcentage exact d'infestation, compte tenu de la difficulté de séparer, sauf par un examen à un très fort grossissement, les formes larvaires des deux espèces (4). Le taux d'infestation de ces Cyclops a certainement dépassé cependant $50 \%$.

$\mathrm{Au} 3^{\mathrm{e}}$ jour : l'onchosphère semble ne pas avoir encore évolué et présente la morphologie qu'elle avait dans l'œuf à maturité $(22 \mu$ sur de 18 à $20 \mu)$. Les crochets embryonnaires sont encore bien à leur place.

Au $16^{\mathrm{e}}$ jour : chez un Cyclops déjà infecté par deux larves de Protéocéphale de corégone, une larve de $P$. macrocephalus mesure $215 \mu$ sur $60 \mu$ y compris le cercomère séparé du reste du corps par un simple étranglement et mesurant à lui seul $45 \mu$ sur $45 \mu$. Pas de ventouses, ni de système excréteur visibles. Les six crochets tous encore dans la partie postérieure. Début de spinulation cuticulaire. Dans le parenchyme, apparition de quelques amas de très petites granulations calcaires.

$\mathrm{Au} 40^{\circ}$ jour: une larve mesure $400 \mu$ sur $150 \mu$, pour laquelle nous n'avons pas retrouvé trace de cercomère, même détaché dans la cavité générale du Copépode (5). Spinulation cuticulaire nette. Canaux excréteurs et vésicule excrétrice nets. Six (peutêtre plus) groupements de trois cellules-flammes visibles. Ventouses principales : $45 \mu$. $5^{\text {e }}$ ventouse bien développée et mobile de $23 \mu$.

- Autre lot en provenance de Lavau (Loire-Atlantique). 65 à $70 \%$ d'infestation. De 1 à 3 larves par Copépode.

$\mathrm{Au} 4^{\circ}$ jour : début de l'évolution de l'onchosphère, dont trois exemplaires chez un même Copépode mesurent respectivement $50 \mu$ sur $45 \mu, 45 \mu$ sur $42 \mu$ et $35 \mu$ sur $30 \mu$. Les crochets embryonnaires commencent à s'éparpiller dans le parenchyme.

(2) En ce qui concerne la détermination de cette espèce et de Cyclops abyssorum en provenance de Thonon, se reporter aux précisions données dans une note antérieure (Jarecka et Doby, 1965).

(3) Le Corégone qui hébergeait ce Protéocéphale à l'état adulte a été déterminé dans la note précédente Coregonus fera. Depuis, nous avons pu obtenir des précisions sur la position spécifique exacte de ce poisson qui, pour certains spécialistes, serait en fait, non la Fera elle-même, mais un hybride entre la fera, pratiquement disparue de nos jours du Lac Léman, et un Corégone introduit relativement récemment en provenance du Lac de Neuchâtel, la Palée. Il n'est pas dans notre propos de vouloir discuter ici d'une détermination à propos de laquelle les spécialistes de poissons euxmêmes semblent n'être pas toujours en accord. Nous remercions vivement M. le Professeur Baer, de Neuchâtel, qui a attiré notre attention sur ce petit problème taxonomique.

(4) Les deux espèces sont cependant relativement facilement reconnaissables par la morphologie de la partie postérieure de l'appareil excréteur, ainsi d'ailleurs que par la taille des crochets embryonnaires: de 7,5 à $10 \mu$ pour $P$. macrocephalus et de 12 à $14 \mu$ pour le Protéocéphale de Corégone.

(5) Au cours de notre travail sur le cycle évolutif d'un Protéocéphale de Corégone, nous avons signalé la survie pendant plusieurs semaines du cercomère détaché dans la cavité générale du Copépode, parfois très loin de la larve elle-même. 
$\stackrel{8}{\circ}$

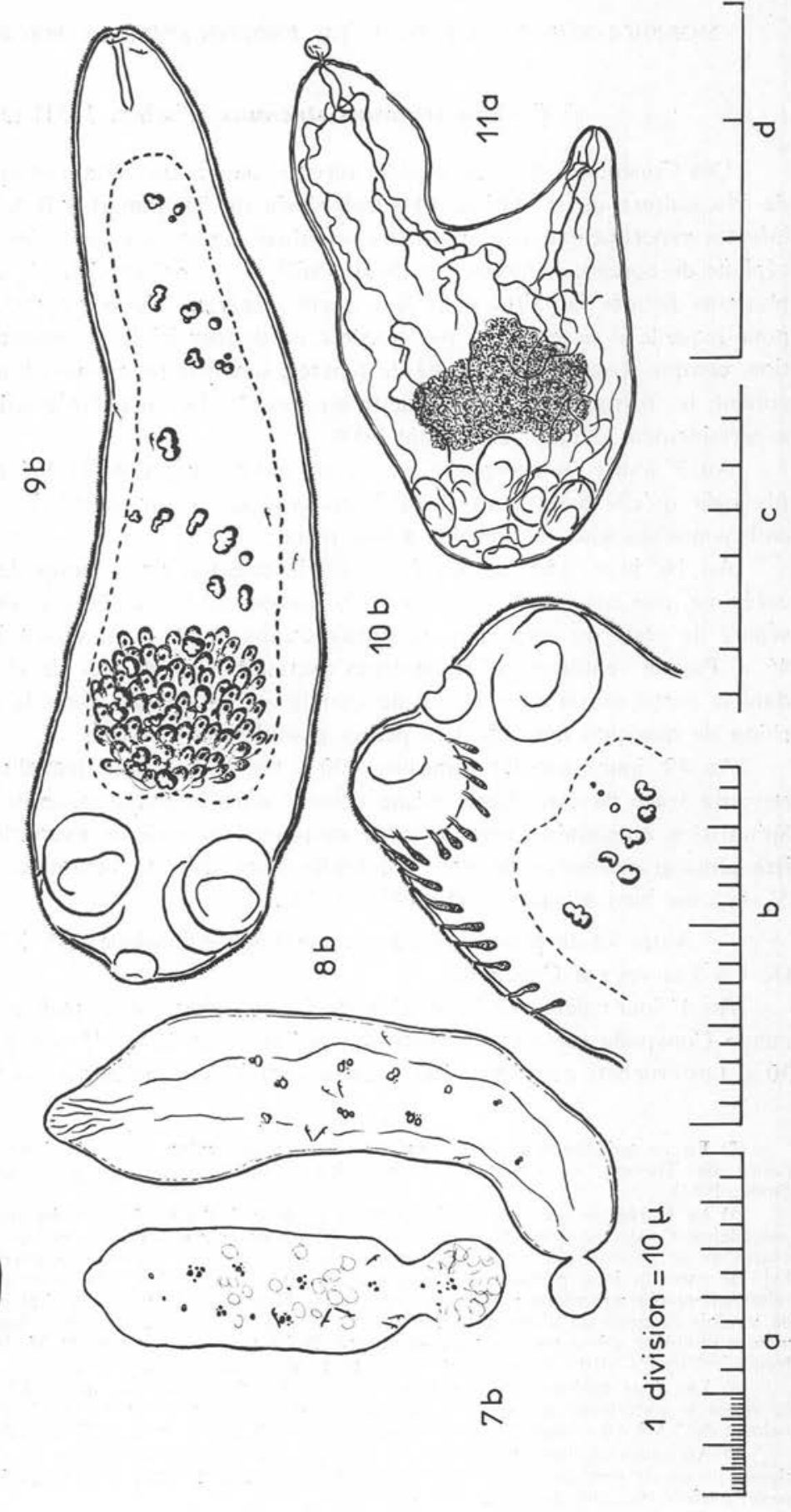

i 8

ㄱ

m 场
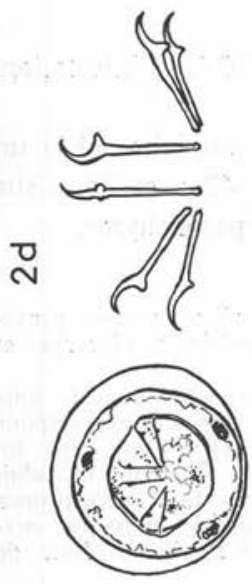

$\because$ 
Au $15^{\circ}$ jour : chez un Copépode, une larve, unique, mesure $410 \mu$ sur $100 \mu$. V.p. : $40 \mu$. Pas de $5^{\circ} \mathrm{V}$. visible. Cercomère retrouvé isolé dans la furca du Copécode, bien mobile, de $40 \mu$ sur $20 \mu$.

$\mathrm{Au} 16^{\circ}$ jour, chez un Copécode, 4 larves mesurent chacune environ $450 \mu$ sur $140 \mu$. V.p. : $45 \mu$. $5^{\circ}$ V. : $23 \mu$, bien musclée, creusée et semblant fonctionnelle ; spinulation cuticulaire et système excréteur nets. Nombreuses granulations calcaires de $10 \mu$ à $15 \mu$ de diamètre.

Au $43^{\circ}$ jour : larve unique de $650 \mu$ sur $150 \mu$.

Au $57^{\circ}$ jour: larve unique de $440 \mu$ sur $110 \mu$. V.p. : $40 \mu .5^{\circ}$ V. : $20 \mu$. Persistance du cercomère, mais aspect particulier de celui-ci, qui, avec une structure très lâche, semble vésiculé. Il mesure $100 \mu$ sur $55 \mu$.

Dans un Cyclops du même élevage examiné dans les mêmes délais a été observé un monstre en partie double constitué d'une partie antérieure unique, mais présentant cinq ventouses principales, dont une nettement plus grosse de $100 \mu$ de diamètre, ainsi que la $5^{\circ}$ ventouse, et une partie postérieure bifide, avec deux systèmes excréteurs et deux vésicules, mais avec un cercomère d'un seul côté de $50 \mu$ de diamètre. Cette larve mesurait $1.000 \mu$ sur $600 \mu$.

Deux crochets embryonnaires étaient inclus dans le cercomère.

\section{b) Cyclops sp. (forme de strenuus ?)}

En provenance de la Brière (Loire-Atlantique). $100 \%$ d'infestation. De deux à quatre formes larvaires par Copépode.

Pas d'évolution suivie des larves pour cette espèce de Copépode qui n'a pu être déterminée.

\section{c) Cyclops abyssorum (Sars, 1863)}

En provenance du Lac Léman près de Thonon. Déjà infesté, comme les Cyclops strenuus strenuus de Thonon, par des larves de Protéocéphale de corégone. Au moins $50 \%$ d'infestation.

1. - Euf à maturité avec son onchosphère et ses trois membranes. 2. - Crochets de l'embryon hexacanthe. $3,4,5,6,7,8,9$. - Différents stades de l'évolution de la forme larvaire dans la cavité générale des Copépodes (du $1^{\mathrm{er}}$ au $40^{\mathrm{e}}$ jour). - Sur les schémas 7 et 8 est figurée la totalité des granulations calcaires existant alors. Par contre, dans le schéma 9, seules quelques granulations calcaires sont figurées. En réalité, sur deux plans différents, elles occupent toute la surface délimitée par le pointillé. - Sur ce même schéma n'est pas figuré le système excréteur; par contre, est figudé le massif des cellules granuleuses de la partie antérieure ainsi que les six crochets embryonnaires dont deux se trouvent à l'extrémité postérieure de la larve. 10. - Partie antérieure d'une forme larvaire à maturité présentant de nombreuses glandes cuticulaires. 11. Larve monstrueuse bifide, avec cinq ventouses (non compris la « $5^{\mathrm{e}}$ ventouse $)$, et deux vésicules excrétrices. Un seul cercomère a été retrouvé. Est figurée également sur ce schéma la zone des cellules granuleuses ainsi que le système des canaux excréteurs particulièrement complexe.

Note : Ces schémas présentent des irrégularités et des asymétries. La raison en est qu'ils ont été dessinés à frais à la chambre claire et n'ont été l'objet d'aucune retouche ni schématisation. Chaque numéro de schémas est accompagné d'une lettre qui renvoie aux diverses échelles micrométriques qui les accompagnent. 
Au $18^{\circ}$ jour : chez un Copépode déjà infecté par quatre larves de Protéocéphale de corégone, deux larves de $P$. macrocephalus mesurent respectivement $160 \mu$ sur $190 \mu$ et $220 \mu$ sur $75 \mu$. V.p. : $30 \mu$. $5^{\circ}$ V. : $20 \mu$, déjà bien formée. Pas de canaux excréteurs, ni vésicule visibles. Mais cellules-flammes dans la partie médiane (15 visibles de chaque côté). Cuticule encore mal délimitée, mais spinulation fine déjà visible sur au moins les deux tiers antérieurs. Pas de cercomère visible. Granulations encore peu nombreuses, fines et réparties en petits amas.

\section{d) Acanthocyclops vernalis (Fischer, 1853)}

En provenance de Lavau (Loire-Atlantique). $70 \%$ d'infestation. De une à quatre larves par Copépode.

Au $8^{\circ}$ jour: une larve seule chez un Copépode se présente sous forme d'un simp'e massif cellulaire allongé, plus renflé à un bout, de $120 \mu$ sur $60 \mu$ au niveau le plus large, avec les six crochets embryonnaires encore groupés à l'extrémité la plus effilée. Pas de cuticule, ni ventouse, ni système excréteur différenciés.

Au $15^{\circ}$ jour : une larve unique mesure $490 \mu$ sur $130 \mu$. V.p. : $50 \mu .5^{\circ}$ ventouse : bien développée, $20 \mu$. Cuticule bien différenciée couverte de fines épines $(0,3$ à $0,5 \mu$ de longueur) sur toute sa surface. Granulations calcaires abondantes (15 $\mu$ pour les plus grosses). Crochets embryonnaires répartis deux de chaque côté dans la partie médiane de la larve et deux dans la partie la plus postérieure. Pas de cercomère visible. Système excréteur bien développé. Vésicule excrétrice avec replis musculeux longitudinaux. 7 ou 8 systèmes de chaque côté de 3 cellules-flammes (à moins qu'il ne s'agisse de 7 systèmes de chaque côté plus un médian postérieur terminal).

$\mathrm{Au} 18^{\circ}$ jour: pas de différences sensibles avec la larve précédente.

Au $22^{\circ}$ jour : chez un Copépode, trois larves mesurent respectivement $350 \mu$ sur $80 \mu$ (deux) et $320 \mu$ sur $120 \mu$. Elles sont retrouvées avec leur cercomère encore fixé, respectivement de $30 \mu$ sur $30 \mu$, de $15 \mu$ sur $15 \mu$ et de $12 \mu$ sur $13 \mu$. V.p. : $40 \mu .5^{\circ}$ V.: $20 \mu$. Même disposition des crochets embryonnaires résiduels que précédemment (6). Dans la zone médiane de ces larves, massif de grosses cellules granuleuses.

Au $35^{\circ}$ jour: deux larves dans un Copépode mesurent $300 \mu$ sur $140 \mu$. V.p. : $50 \mu .5^{\circ}$ V.: $30 \mu$. Granulations calcaires nombreuses et volumineuses de $15 \mu$ à $18 \mu$ sur deux plans longitudinaux séparés. Un cercomère $(25 \mu$ sur $25 \mu)$ retrouvé séparé dans la furca du Copépode.

\section{e) Diacyclops bicuspidatus (Claus, 1857)}

En provenance de Lavau (Loire-Atlantique). $10 \%$ d'infestation. De une à trois larves par Copépode.

(6) En règle générale, les crochets embryonnaires résiduels s'observent chez la larve de $P$. macrocephalus en une répartition beaucoup plus régulièrement définie que chez les larves au même stade du Protéocéphale de Corégone que nous avons pu étudier au cours d'un travail antérieur. 
Au $13^{\circ}$ jour: larve unique de $340 \mu$ sur $85 \mu$, y compris un cercomère bien marqué de $27 \mu$ de diamètre. Pas de ventouses, ni canaux excréteurs, ni vésicule, ni spinulation cuticulaire visibles. Crochets embryonnaires encore dans la partie médiane. Quelques rares granulations calcaires de 2 à $5 \mu$.

Au $50^{\circ}$, jour : trois larves chez un même Copépode mesurent respectivement $950 \mu$ sur $200 \mu$ et $700 \mu$ sur $250 \mu$ (deux). V.p. : $70 \mu$. $5^{\circ}$ V. : $40 \mu$. Aucun cercomère n'est retrouvé.

f) Diaptomus castor (Jurine, 1820)

En provenance de Lavau (Loire-Atlantique). $60 \%$ environ d'infestation. De une à cinq larves par Copépode.

$\mathrm{Au} 13^{\circ}$ jour, deux larves chez un Copépode mesurent $400 \mu$ sur $120 \mu$. V.p. : $60 \mu$, mais mal différenciée. Pas de $5^{\circ} \mathrm{V}$. visible. Granulations calcaires nombreuses de $10 \mu$. Canaux excréteurs et vésicule nets; spinulation très faiblement visible. Présence de deux crochets dans la partie postérieure. Pas de cercomère retrouvé.

$\mathrm{Au} 32^{\circ}$ jour : chez un Copépode, trois larves mesurent respectivement $500 \mu$ sur $140 \mu, 750 \mu$ sur $200 \mu$ et $800 \mu$ sur $210 \mu$. Chez la plus grande, V.p. de $60 \mu$. $5^{\circ} \mathrm{V}$. de $30 \mu$, fonctionnelle. Peuvent ici être observées de nombreuses glandes cuticulaires, surtout dans la partie antérieure de la larve et débouchant sur le pourtour des ventouses. Ces glandes cuticulaires, unicellulaires semble-t-il, mesurent $20 \mu$ de long sur 4 à $5 \mu$ de diamètre maximum et ont un contenu granuleux.

Les différences des pourcentages d'infestation observées entre les trois espèces, Acanthocyclops vernalis, Diacyclops bicuspidatus et Diaptomus castor (respectivement $70 \%, 10 \%$ et $60 \%$ ) sont particulièrement intéressantes et sont peut-être en relation avec une « réceptivité » liée à l'espèce, puisque ces Copépodes, en provenance du même biotope, ont été mis ensemble en contact avec les œufs de Protéocéphale et ont eu par conséquent les mêmes chances de s'infester.

\section{Commentaires}

De ces observations, il résulte :

$1^{\circ}$ que la présence de larves d'une espèce de Protéocéphale chez un Copépode n'empêche pas la surinfestation par des larves d'une autre espèce de Protéocéphale. Ainsi, chez Cyclops strenuus strenuus et Cyclops abyssorum, la présence parfois de plusieurs larves de Protéocéphale de corégone déjà à maturité n'a pas empêché l'infestation par des larves de Protéocéphale d'anguilles, ni l'évolution ultérieure de celles-ci jusqu'à la totale maturité.

$2^{\circ}$ D'une espèce de Copépode à l'autre, comme cela a déjà été dit plus haut, des différences considérables dans la vitesse moyenne d'évolution des larves peuvent être observées. Ainsi, si, compte tenu du petit nombre des larves mensurées, il n'est pas 
possible de trouver de différences significatives pendant les premiers jours de l'évolution d'une espèce de Copépode à l'autre, par contre, dans les semaines qui suivent, des différences nettes apparaissent, par exemple entre les larves parasites de Cyclops strenuus de Lavau et Acanthocyclops vernalis d'une part et celles parasites de Diaptomus castor et Diacyclops bicuspidatus d'autre part. Chez ces derniers en effet, les larves sont plus de deux fois plus grosses que chez les premiers aux environs du $50^{\circ}$ jour.

$3^{\circ}$ Chez des Copépodes de la même espèce infestés de la même façon, tenus en élevage commun et parasités par un même nombre de larves, on peut observer d'un Copépode à l'autre de grosses différences d'évolution. Par exemple, chez un Cyclops strenuus de Lavau, une larve unique, 15 jours après l'infestation, mesurait $410 \mu$ sur $100 \mu$, alors que chez un autre Copépode de la même série expérimentale, une larve, également unique, 57 jours après l'infestation, ne mesurait que $440 \mu$ sur $110 \mu$.

Ces deux constatations montrent la valeur très relative qu'il convient d'attribuer à des dimensions larvaires de larves pourtant à maturité trouvées dans la nature.

$4^{\circ}$ Enfin, il semble qu'il ne soit pas possible d'établir de relations entre la taille de la larve et l'état de maturation de ses différents éléments morphologiques (spinulation cuticulaire, ventouses, système excréteur, etc...).

Ainsi, il est intéressant de comparer la morphologie des larves ci-dessous pourtant d'âges très voisins :

a) Chez une larve de 18 jours chez Cyclops abyssorum : $220 \mu$ sur $75 \mu$. Les cinq ventouses déjà bien discernables. Spinulation cuticulaire nette. Cellules-flammes visibles. Cercomère déjà disparu. Par contre, granulations calcaires encore peu nombreuses, fines et réparties en petits amas.

b) Larve de 13 jours chez Diacyclops bicuspidatus: $340 \mu$ sur $85 \mu$. Pas de ventouses, ni système excréteur, ni spinulation cuticulaire visible. Cercomère encore fixé. Très rares granulations calcaires fines.

c) Larves de 13 jours chez Diaptomus castor : $400 \mu$ sur $120 \mu$. Ventouses principales discernables, mais pas la $5^{\circ}$. Système excréteur bien développé. Spinulation cuticulaire nette. Pas de cercomère visible. Granulations calcaires nombreuses atteignant un diamètre de $10 \mu$.

d) Larves de 15 jours chez Acanthocyclops vernalis : $490 \mu$ sur $130 \mu$. Ventouses bien développées, y compris la $5^{\circ}$. Cuticule tout à fait différenciée avec spinulation. Système excréteur complet notamment avec l'ensemble des cellules-flammes et vésicule excrétrice à paroi musculeuse. Granulations calcaires abondantes dont les plus volumineuses atteignent $15 \mu$ de diamètre. 


\section{Conclusions}

Dans les conditions expérimentales où nous avons opéré, il faut un minimum de 15 jours entre l'ingestion des œuf́s et l'état de maturité de la larve de Protéocéphale dans la cavité générale, cette maturation pouvant, compte tenu des variations dans les vitesses d'évolution citées plus haut, demander beaucoup plus longtemps. La majorité des larves arrivent cependant à leur maturité, semble-t-il, dans des délais variant entre 20 et 30 jours après l'infestation.

\section{Essais d'infestation d'Hôtes définitifs}

Avec ces Copépodes infestés, nous avons pu tenter l'infestation de diverses espèces de poissons. Pour toutes ces tentatives, ont seuls été utilisés des Copépodes infectés au moins depuis 30 jours, c'est-à-dire renfermant dans leur cavité générale des larves ayant dans leur majorité statistiquement atteint leur maturité.

\section{Essais négatifs :}

$1^{\circ}$ Un jeune poisson-chat [Amiurus nebulosus (Lesieur)], d'une taille de $5 \mathrm{~cm}$, a été trouvé négatif 27 jours après avoir été mis en contact avec plusieurs Cyclops strenuus infestés.

$2^{\circ}$ Deux perches du Canada [Eupomotis gibbosus (Lin.)] et deux perches ordinaires (Perca fluviatilis Lin.) ont été utilisées. Un exemplaire de chaque espèce a été simplement mis en présence de Copépodes infestés, un exemplaire de chaque espèce a été infecté par ingestion contrôlée d'un ver de terre dans les tissus duquel avaient été inclus, pour chaque, 30 Cyclops strenuus infectés chacun avec de une à trois larves de Protéocéphale.

Un individu de chaque espèce a été trouvé négatif respectivement 3 jours et 13 jours après la tentative d'infestation.

$3^{\circ}$ Deux cyprinidés sp. (gros alevins), mis simplement en contact avec des Copépodes infectés, ont été trouvés négatifs 165 jours après l'infestation.

\section{Infestations positives :}

18 anguilles mesurant environ $15 \mathrm{~cm}$ de long, sauf une de $22 \mathrm{~cm}$, récoltées plusieurs mois au préalable à l'état de civelles sur les bords de la Loire et élevées depuis en laboratoire avec de la nourriture artificielle, furent infestées par simple mise en contact avec les Copépodes infectés. Furent utilisés pour cette tentative des Cyclops strenuus et Acanthocyclops vernalis en provenance de Lavau. Ce contact eut lieu avec un minimum par anguille de cinq Copépodes infestés depuis au moins 20 jours 
et renfermant chacun de une à quatre larves à maturité. Ces anguilles furent sacrifiées respectivement 10,13,22, 30, 38, 45 (2), 70 (2 dont celle de $22 \mathrm{~cm}$ ), 90 (2), 120 (4) et 150 (3) jours après le contact.

Des larves de Protéocéphale furent retrouvées régulièrement dans toutes les anguilles sacrifiées jusqu'au $45^{\circ}$ jour. Les anguilles examinées ultérieurement furent trouvées négatives. Les positives hébergeaient respectivement six larves $\left(10^{\circ}\right.$ jour), six $\left(13^{\circ}\right)$, cinq $\left(22^{\circ}\right)$, deux $\left(30^{\circ}\right)$, cinq $\left(38^{\circ}\right)$, quatre et deux $\left(45^{\circ}\right)$.

Voici la description de quelques-unes des formes larvaires retrouvées dans l'intestin de ces anguilles :

$1^{\circ}$ Chez des anguilles infectées avec Cyclops strenuus :

- au $10^{\circ}$ jour: larve de $520 \mu$ sur $160 \mu$. V.p. de $50 \mu$. $5^{\circ}$ V.: $30 \mu$. Crochets embryonnaires encore retrouvés. Nombreuses granulations calcaires persistant ;

- au $22^{\circ}$ jour : trois des cinq larves trouvées présentent respectivement les mensurations et caractères suivants :

- $520 \mu$ sur $200 \mu$. V.p. : $60 \mu .5^{\circ}$ V.: $20 \mu$,

- $750 \mu$ sur $300 \mu$. V.p. : $65 \mu .5^{\circ}$ V. : $24 \mu$,

- $1.000 \mu$ sur $350 \mu$. V.p. : $80 \mu .5^{\circ}$ V.: $30 \mu$.

Il persiste encore de grosses granulations calcaires, mais moins nombreuses que dans les larves chez le Copépode. Par contre, dans le parenchyme de toute la moitié postérieure de la larve, de très nombreuses très petites granulations réfringentes sont observées, réparties d'une façon homogène. Les crochets embryonnaires n'ont pu être retrouvés. Le système excréteur devient beaucoup plus complexe, notamment dans l'organisation des canaux secondaires.

Même chez le dernier exemplaire, dont les ventouses ont presque atteint les dimensions de celles du Cestode adulte $(90$ à $100 \mu$ pour les V.p. et $40 \mu$ pour la $5^{\circ} \mathrm{V}$.), aucune trace de segmentation n'est visible (7).

$2^{\circ}$ Chez des anguilles infectées avec Acanthocyclops vernalis :

- au $13^{\circ}$ jour, deux des six larves retrouvées présentent les caractéristiques suivantes :

- $400 \mu$ sur $140 \mu$,

- $640 \mu$ sur $200 \mu$. V.p. : $60 \mu$. Pas de $5^{\circ}$ V. nette. Granulations calcaires larvaires semblant en voie de disparition car beaucoup plus dispersées, moins nombreuses et plus petites que chez les larves observées chez les Copépodes. Par contre, le système excréteur semble, ici aussi, être devenu plus complexe. Chez ces deux exemplaires, les crochets embryonnaires n'ont pu être retrouvés, même après examen systématique au fort grossissement après aplatissement à la limite de l'écrasement.

(7) Le développement et les dimensions de la $5^{\circ} \mathrm{V}$. chez cet exemplaire de $1.000 \mu$ nous permet d'émettre des doutes quant à la détermination comme Proteocephalus macrocephalus par Soltynska (1964) d'un jeune Cestode trouvé chez l'anguille, "sans $5^{\circ}$ ventouse» visible, bien qu'il se soit agi d'un exemplaire dont la taille atteignait déjà $2.000 \mu$ de long. 
Par la suite, chez les larves retrouvées jusqu'au $45^{\circ}$ jour, nous n'avons pu observer ni augmentation de taille ni modifications morphologiques importantes, notamment une amorce de segmentation.

\section{Commentaires}

Les faits suivants ressortent de notre expérimentation:

$1^{\circ}$ Il semble exister une limitation du nombre de parasites pouvant se développer dans la lumière de l'intestin de l'anguille, puisque le maximum de larves observées a été de six, alors que les infestations étaient généralement lourdes. Ainsi, par exemple, certaines anguilles infectées avec 20 Cyclops strenuus infestés à raison de $70 \%$ avec d'une à trois larves chacune, ont donc reçu en moyenne statistiquement environ 30 larves de Protéocéphale. Or, ces anguilles ont permis de retrouver 6 et 5 larves, respectivement 10 et 22 jours après le repas infestant.

$2^{\circ}$ L'évolution des formes larvaires semble être assez lente, du moins pendant les premiers temps de l'infestation des anguilles et dans nos conditions expérimentales, puisque les larves que nous avons retrouvées chez celles-ci, jusqu'au $45^{\circ}$ jour, ne diffèrent pas tellement par leur taille des plus grands exemplaires rencontrés chez les Copépodes. Les principales différences observées portent sur une taille plus grande des ventouses, la disparition partielle des grosses granulations calcaires larvaires et la complication progressive du système excréteur dont les ensembles de cellules-flammes s'enrichissent de canaux secondaires anastomosés. Nous avons pu parfois observer en outre, dans la zone postérieure à la région des cellules granuleuses, six formations longitudinales (vraisemblablement ébauches de faisceaux musculaires).

$3^{\circ}$ Nous avons observé, assez rapidement, l'élimination spontanée des parasites, puisque nous n'avons pas retrouvé une seule larve de Cestode chez les onze anguilles sacrifiées au $70^{\circ}$ jour et après. Il ne nous est pas possible de donner les raisons de cette élimination des parasites. Cela est peut-être dû aux conditions d'élevage des anguilles (8), au type de nourriture notamment.

Comme raison de cet échec peut être envisagée également l'existence d'un deuxième hôte intermédiaire obligatoire, mais cette existence est peu vraisemblable, si l'on se réfère aux cycles de Protéocéphales déjà connus d'une part, d'autre part au résultat négatif que nous avons obtenu dans nos tentatives d'infestation d'espèces de poissons autres que les anguilles, même lorsque l'examen était très précoce après le repas infestant. Enfin, l'espèce de Protéocéphale ici en cause se retrouve à l'état adulte normalement et uniquement chez l'anguille dans la nature.

(8) Dans une lettre récente, M. le Professeur Baer nous a fait part d'observations analogues, en laboratoire, d'élimination spontanée des parasites effectuées chez des alevins de Corégones pourtant infestés avec des larves appartenant à l'espèce de Protéocéphale qui leur est habituellement parasite. 


\section{Bibliographie}

DoBy (J.-M.) et JARECKA (L.), 1964. - Redescription d'un Proteocephalus (Cestode) parasite du poisson Coregonus fera, en provenance du Lac Léman. Problèmes posés par la diagnose spécifique des Cestodes du genre Proteocephalus. Bull. Soc. Zool. de France, 89, 675.

JARECKA (L.), 1960. - Life-cycles of tapeworms from lakes Goldapiwo and Mamry Polnocne. Acta Parasit. Polonica, 8, 47.

JARecka (L.) et Doby (J. M.), 1965. - Contribution à l'étude du cycle évolutif d'un Cestode du genre Proteocephalus parasite de Coregonus fera, en provenance du Lac Léman. Ann. Parasit. hum. et comp. 40, 433.

Joyeux (C.) et BAer (J. G.), 1936. - Cestodes. Faune de France, Paris, Lechevalier éd.

LA RUE (G.-R.), 1911. - A revision of the Cestode family Proteocephalidae, Thèse de Doctorat de l'Univ. de l'Illinois. In Illinois biological monographs, $1, \mathrm{n} .1$ et 2 .

Markowski (S.), 1933. - Die Eingeweidewürmer der Fische des polnischen Balticums. Arch. Hydrobiol. i. Rybactwa, 7.

SCHNEIDER (G.), 1905. - Die Ichthyotaenien des finnischen Meerbusens. Festschr. Palmen, Helsingfors, 1,1 .

SołtynSKa (M.), 1964. - Fish tapeworms in Puck Bay (South Baltic). Acta Parasit. Polonica, 12, 13.

WARdLe (R. A.) et Mc LeOd (J. A.), 1952. - The Zoology of Tapeworms, North central Publ. Co., St-Paul, Minnesota.

Yamaguti (S.), 1959. - Systema helminthum, vol. 2 (The Cestodes of Vertebrates), New York, Intersc. Publ.

(Laboratoire de Parasitologie et Zoologie appliquée de la Faculté de Médecine et de Pharmacie de Rennes) 